\title{
Quantum effects in the Brownian motion of a particle in a double well potential in the overdamped limit
}

\author{
William T. Coffey, ${ }^{1}$ Yuri P. Kalmykov, ${ }^{2, a)}$ Serguey V. Titov, ${ }^{3}$ and Liam Cleary ${ }^{1}$ \\ ${ }_{1}^{1}$ Department of Electronic and Electrical Engineering, Trinity College, Dublin 2, Ireland \\ ${ }^{2}$ Laboratoire de Mathématiques, Physique et Systèmes, Université de Perpignan, 52, Avenue de Paul Alduy, \\ 66860 Perpignan Cedex, France \\ ${ }^{3}$ Institute of Radio Engineering and Electronics, Russian Academy of Sciences, Vvedenskii Square 1, \\ Fryazino, 141190, Russia
}

(Received 22 June 2009; accepted 2 August 2009; published online 24 August 2009)

\begin{abstract}
Quantum effects in the noninertial Brownian motion of a particle in a double well potential are treated via a semiclassical Smoluchowski equation for the time evolution of the reduced Wigner distribution function in configuration space allowing one to evaluate the position correlation function, its characteristic relaxation times, and dynamic susceptibility using matrix continued fractions and finite integral representations in the manner of the classical Smoluchowski equation treatment. Reliable approximate analytic solutions based on the exponential separation of the time scales of the fast intrawell and slow overbarrier relaxation processes are given. Moreover, the effective and the longest relaxation times of the position correlation function yield accurate predictions of both the low and high frequency relaxation behavior. The low frequency part of the dynamic susceptibility associated with the Kramers escape rate behaves as a single Lorentzian with characteristic frequency given by the quantum-mechanical reaction rate solution of the Kramers problem. As a particular example, quantum effects in the stochastic resonance are estimated. (C) 2009 American Institute of Physics. [DOI: 10.1063/1.3211021]
\end{abstract}

\section{INTRODUCTION}

The Brownian motion in the double well potential,

$$
V(x)=\frac{1}{2} a x^{2}+\frac{1}{4} b x^{4}, \quad-\infty<x<\infty,
$$

where $a$ and $b$ are constants, is ubiquitous in the modeling of diverse physical problems involving bistability and noise activated transitions between the minima of a bistable potential. Examples are simple isometrization processes, chemical reaction rate theory, bistable nonlinear oscillators, second order phase transitions, and nuclear fission and fusion (see, e.g., Refs. 1-5 and references cited therein).

Now the dynamics of a classical Brownian particle in the double well potential in the very high damping (VHD) limit, where the inertia of the particle may be neglected, have been extensively studied using the Smoluchowski equation for the evolution of the distribution function in configuration space (see, e.g., Refs. 6-10) because that equation essentially circumvents the mathematical difficulties associated with the exact phase space treatment based on the Fokker-Planck equation. The Smoluchowski equation governs the time behavior of classical point Brownian particles in configuration space if the energy loss per cycle of the almost periodic motion of a particle moving along a saddle point of the potential (that is, on an escape trajectory in phase space) is much greater than the thermal energy. The high damping condition is tantamount to assuming in the Fokker-Planck equation for the joint distribution of the positions and momenta of the particle in phase space $(x, p)$ that the momen-

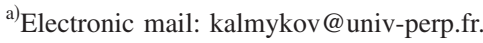

tum $p$ has reached the equilibrium Maxwellian distribution, while the position $x$ has not yet had sufficient time to reach the Boltzmann distribution. The particular Fokker-Planck equation pertaining to the inertial motion of point Brownian particles with separable and additive Hamiltonians is known as the Klein-Kramers equation. The analysis of quantum effects in the noninertial Brownian motion in a double well potential, which is the main topic of this paper, therefore requires a semiclassical generalization of the Smoluchowski equation. This is possible using Wigner's reformulation ${ }^{1-13}$ of quantum mechanics as a statistical theory on classical phase space because that representation allows one to construct a master equation for the evolution of the quasiprobability distribution of positions and momenta of a quantum Brownian particle akin to the Fokker-Planck equation. Hence, the desired quantum Smoluchowski equation in configuration space, which is valid in the high bath-particle dissipation limit, may be derived. ${ }^{14-17}$

In general Wigner's phase space representation allows one to calculate nonequilibrium quantum mechanical averages involving the time dependent density matrix by integration over the distribution function in the classical manner using the Weyl symbol of a quantum operator. ${ }^{18}$ This is accomplished $^{14}$ via the phase space master equation by representing in the manner of the classical Fokker-Planck equation the collision term (which takes account of the interchange of energy between a particle and its heat bath) by a Kramers-Moyal expansion ${ }^{4}$ truncated at the second order. The Kramers-Moyal coefficients in this expansion are then calculated by postulating, instead of the Maxwell-Boltzmann distribution, the Wigner equilibrium distribution in phase 
space as the stationary solution. The foregoing ansatz (essentially representing an extension of Einstein's method ${ }^{4}$ of determining diffusion coefficients in Fokker-Planck equations) leads to the desired semiclassical master equation for the time evolution of the quasidistribution function in phase space. Now, the master equation may be solved to any order in Planck's constant $\hbar$ via perturbation theory by first reducing it to a partial differential-recurrence relation in configuration space. This set of equations is obtained by expanding the momentum part of the distribution function in orthogonal Hermite polynomials ${ }^{19}$ so generating a quantum generalization of Brinkman's well known representation ${ }^{20,21}$ of the Klein-Kramers equation as a set of time-dependent partial differential-recurrence equations in configuration space. The recurrence equations, known in the classical case ${ }^{21}$ as the Brinkman equations, may then be further reduced (if the form of the potential is prescribed so that an appropriate orthogonal expansion of the spatial part of the distribution function may be made) to a set of ordinary differential recurrence equations for the statistical moments (observables), which may be solved to any order of perturbation theory in $\hbar$ in the frequency domain by matrix continued fraction methods. $^{22-24}$

However, since the emphasis here is on quantum effects in the overdamped limit, the solution may be drastically simplified by means of the quantum Smoluchowski equation, which holds (just as its classical counterpart) if the energy loss per cycle of particles on the escape trajectory is much greater than the thermal energy. The quantum Smoluchowski equation (again just as its classical counterpart) relies on the assumption that the momentum part of the phase space distribution has reached equilibrium long before the configuration part and has been presented in Refs. 14 and 15. Following arguments used by Kramers ${ }^{25}$ to derive the classical Smoluchowski equation from the Klein-Kramers equation, this is accomplished by integrating the phase space master equation, to first order in perturbation theory, over a straight line in phase space, and then proceeding to the noninertial (overdamped) limit assuming that the equilibrium distribution of the momenta has set in. The procedure then leads to an equation of essentially the same form as the classical Smoluchowski equation. However, the diffusion coefficient now becomes a function of the derivatives of the potential while the drift coefficient remains unaltered. The same conclusion may be drawn by constructing from the phase space master equation the quantum recurrence equations in configuration space to the first order of perturbation theory and then proceeding to the noninertial limit (Brinkman's method). However, a much simpler albeit indirect method $^{14,15}$ of proceeding (prompted by the form of the first order perturbation solution) is to postulate a priori to any order of perturbation theory the Smoluchowski equation with a position dependent diffusion coefficient. This coefficient is then calculated in terms of the derivatives of the potential by imposing as the stationary solution the Wigner equilibrium configuration space distribution, which may be calculated from the stationary phase space distribution by elementary integration over the momenta. The method then yields the quantum Smoluchowski equation to any desired order in
Planck's constant. In contrast, in this paper, by way of reinforcement of the preceding arguments, we shall show how that equation in the second order (and thus to any order) of perturbation theory may be derived directly from the quantum Brinkman equations generated by the phase space master equation by proceeding to the noninertial limit. Moreover, we shall demonstrate how the existing theory for a Brownian particle in a double well potential may be extended to include quantum effects in semiclassical fashion.

A quantum Smoluchowski equation has been used in many applications of the quantum Brownian motion in a potential. $^{16,26,27}$ Here, having determined the quantum Smoluchowski equation in the manner outlined above, we shall use it to evaluate quantum corrections in the Brownian motion of the particle moving in the double well potential given by Eq. (1). Certain aspects of quantum effects in the Brownian motion in a double well potential have already been analyzed, e.g., in Refs. 24 and 28-32. In particular, we shall estimate quantum effects in the equilibrium position correlation function $C(t)=\beta^{-1}\left\langle\int_{0}^{\beta} \hat{x}(-i \lambda \hbar) \hat{x}(t) d \lambda\right\rangle_{0}$, its spectrum and characteristic relaxation times, dynamic susceptibility, and stochastic resonance by solving the quantum Smoluchowski equation using the matrix continued-fraction methods already developed for the classical problem. ${ }^{5}$ The symbol $\langle\cdots\rangle_{0}$ denotes equilibrium ensemble averages, $\beta$ $=(k T)^{-1}, k$ is Boltzmann's constant and $T$ is the temperature. The calculations will illustrate how one may evaluate observables in the familiar classical manner. In particular, we shall demonstrate that the single mode approximation for the correlation function spectrum yields closed analytic solutions for the dynamic susceptibility at low frequencies. Furthermore, we shall calculate the correlation time and the mean first passage time (MFPT) for a Brownian particle to leave a well of the potential. These times may be determined, just as the classical case, knowing the quantum diffusion coefficient and stationary distribution only.

\section{QUANTUM SMOLUCHOWSKI EQUATION: BRINKMAN'S METHOD}

In the quantum Brownian motion, a semiclassical master equation for the translational Brownian motion of a particle of mass $m$ moving in a potential $V(x)$ can be derived by proceeding to the high temperature limit. This is equivalent to treating a typical Brownian particle as a quantum mechanical particle embedded in a classical bath. ${ }^{33}$ The master equation for the time evolution of the single-particle quasiprobability distribution $W(x, p, t)$ in phase space $(x, p)$ is ${ }^{14,15}$

$$
\begin{aligned}
& \frac{\partial W}{\partial t}+\frac{p}{m} \frac{\partial W}{\partial x}-\frac{1}{i \hbar}\left[V\left(x+\frac{i \hbar}{2} \frac{\partial}{\partial p}\right)-V\left(x-\frac{i \hbar}{2} \frac{\partial}{\partial p}\right)\right] W \\
& =\hat{M}_{D} W
\end{aligned}
$$

where $\hat{M}_{D}$ is the collision kernel operator which accounts for effects due to the coupling of the particle to its environment (dissipation and fluctuations). The left-hand side of this equation is the quantum analog of the classical Liouville equation pertaining to a closed system, where the collision 
term is zero. The stationary solution of this equation [i.e., the master Eq. (2) with the right hand side equal to zero] is the Wigner stationary distribution $W_{0}(x, p)$, which can be developed as a power series in $\hbar^{2}$, viz., ${ }^{13,14}$

$$
\begin{aligned}
W_{0}(x, p)= & e^{-\beta \varepsilon(x, p)}\left\{1+\frac{(\beta \hbar)^{2}}{24 m}\right. \\
& \left.\times\left[\beta V^{\prime 2}(x)-\left(3-\frac{\beta p^{2}}{m}\right) V^{\prime \prime}(x)\right]+\cdots\right\},
\end{aligned}
$$

where $\varepsilon(x, p)=p^{2} /(2 m)+V(x)$ is the classical energy of the particle. The distribution $W_{0}(x, p)$ corresponds to the canonical density matrix $\hat{\rho}_{e q}=e^{\beta \hat{H}} / Z$ ( $\hat{H}$ is the Hamiltonian of the system and $Z$ is the partition function) and describes the system in thermal equilibrium without coupling to the thermal bath, i.e., it pertains to the closed system). Now, on specializing to the quantum Brownian motion in the high temperature and weak coupling limits, the collision kernel operator $\hat{M}_{D}$ can be represented just as in the classical theory by a Kramers-Moyal-like expansion truncated at the second term $^{13,14}$

$$
\hat{M}_{D} W=\frac{\partial}{\partial p}\left[D_{p} p W+D_{p p} \frac{\partial W}{\partial p}+D_{x p} \frac{\partial W}{\partial x}+\cdots\right] .
$$

Here the coefficients $D_{p}, D_{p p}$, and $D_{x p}$ are coordinate, momentum, and time dependent parameters, which are to be determined. Various forms of the collision kernel operator $\hat{M}_{D}$ have been discussed in detail in Ref. 13. In the classical limit $\hbar \rightarrow 0$, the coefficients $D_{p}, D_{p p}$, and $D_{x p}$ become

$$
D_{p}=\gamma, \quad D_{p p}=\gamma m / \beta, \quad D_{x p}=0,
$$

where $\gamma=\zeta / m$ is a dissipation parameter and $\zeta$ is the friction coefficient characterizing the bath-particle interactions, and Eq. (2) reduces $^{14}$ to the Klein-Kramers (Fokker-Planck) equation,

$$
\frac{\partial W}{\partial t}+\frac{p}{m} \frac{\partial W}{\partial x}-\frac{\partial V}{\partial x} \frac{\partial W}{\partial p}=\gamma \frac{\partial}{\partial p}\left(p W+\frac{m}{\beta} \frac{\partial W}{\partial p}\right) .
$$

Unlike the classical theory, in order that the Wigner stationary distribution $W_{0}(x, p)$ should also render the right hand side of Eq. (2) zero, the coefficients $D_{p}, D_{p p}$, and $D_{x p}$ must become functions of the derivatives of the potential. The master Eq. (2) in phase space then describes the relaxation of $W(x, p, t)$ to the stationary state given by $W_{0}(x, p)$ in the long time limit. ${ }^{14}$ We remark that the imposition of $W_{0}(x, p)$ as the stationary solution of Eq. (2) is exactly analogous to the assumption of the Maxwell-Boltzmann distribution as the stationary distribution in the classical Brownian motion. ${ }^{14}$ In Refs. 14 and 15, we evaluated $D_{p}, D_{p p}$, and $D_{x p}$ in the approximation of frequency independent damping, where $D_{p}$, $D_{p p}$, and $D_{x p}$ in Eq. (2) are independent of the time, and have derived the explicit form of Eq. (2) to order $\hbar^{4}$, viz.,

$$
\begin{aligned}
\frac{\partial W}{\partial t}+ & \frac{p}{m} \frac{\partial W}{\partial x}-\frac{\partial V}{\partial x} \frac{\partial W}{\partial p}+\frac{\hbar^{2}}{24} \frac{\partial^{3} V}{\partial x^{3}} \frac{\partial^{3} W}{\partial p^{3}}-\frac{\hbar^{4}}{1920} \frac{\partial^{5} V}{\partial x^{5}} \frac{\partial^{5} W}{\partial p^{5}}+\cdots \\
= & \gamma \frac{\partial}{\partial p}\left[p W+\frac{m}{\beta}\left\{1+\frac{\hbar^{2} \beta^{2}}{12 m} \frac{\partial^{2} V}{\partial x^{2}}-\frac{\hbar^{4} \beta^{4}}{1440 m^{2}}\left[6 \frac{\partial^{3} V}{\partial x^{3}} \frac{\partial V}{\partial x}\right.\right.\right. \\
& \left.\left.\left.+2\left(\frac{\partial^{2} V}{\partial x^{2}}\right)^{2}+3 \frac{\partial^{4} V}{\partial x^{4}}\left(\frac{p^{2}}{m}-\frac{5}{\beta}\right)\right]\right\} \frac{\partial W}{\partial p}\right]+\cdots
\end{aligned}
$$

The master Eq. (3) may be used in the high-temperature approximation, viz., ${ }^{14}$

$$
T \gg T_{0}=\hbar \zeta /(2 \pi m k) \text {. }
$$

This condition is valid in a wide range of model parameters if the interactions between the Brownian particle and the heat bath are small enough to allow one to use the weak-coupling limit and if the correlation time characterizing the bath is so short that we can regard the stochastic process originating in the bath as Markovian. ${ }^{34}$

Following Brinkman's method, ${ }^{15}$ Eq. (3) may be rewritten as a partial differential-recurrence relation in configuration space $x$ by expanding the momentum part of the distribution function, $W(x, p, t)$ in an orthonormal basis of Weber (harmonic oscillator) functions $D_{n}(y), v i z .,{ }^{14}$

$$
W(x, p, t)=e^{-\beta p^{2} / 4 m} \sum_{n=0}^{\infty} D_{n}(p \sqrt{\beta / m}) \varphi_{n}(x, t),
$$

where $D_{n}(y)=2^{-n / 2} e^{-y^{2} / 4} H_{n}(y / \sqrt{2})$ and $H_{n}(z)$ is the Hermite polynomial of order $n .{ }^{19}$ Here the Fourier coefficients $\varphi_{n}(x, t), n \geq 0$ are the configuration space functions. In particular, $\varphi_{0}(x, t)=\int W(x, p, t) d p$ yields the configuration space distribution function. After a tedious calculation using the raising property of the Weber functions, namely,

$$
\frac{d}{d y}\left(e^{-y^{2} / 4} D_{n}(y)\right)=-e^{-y^{2} / 4} D_{n+1}(y),
$$

and the recurrence relation

$$
D_{n+1}(y)=y D_{n}(y)-n D_{n-1}(y),
$$

together with their orthogonality relations, we have ${ }^{14}$ the quantum recurrence equations

$$
\begin{aligned}
\frac{\partial \varphi_{n}}{\partial t}+ & n \gamma\left[1+\frac{6 \Lambda^{2}(n+1)}{5 \beta} \frac{\partial^{4} V}{\partial x^{4}}\right] \varphi_{n} \\
= & -\sqrt{\frac{\beta}{m}} \varphi_{n-1} \frac{\partial V}{\partial x}-\frac{1}{\sqrt{\beta m}}\left(\frac{\partial \varphi_{n-1}}{\partial x}+(n+1) \frac{\partial \varphi_{n+1}}{\partial x}\right) \\
& +\Lambda\left(2 \gamma \varphi_{n-2} \frac{\partial^{2} V}{\partial x^{2}}+\frac{1}{\sqrt{\beta m}} \varphi_{n-3} \frac{\partial^{3} V}{\partial x^{3}}\right) \\
& -\frac{3 \Lambda^{2}}{10 \sqrt{m \beta^{3}}} \varphi_{n-5} \frac{\partial^{5} V}{\partial x^{5}}-\frac{2 \gamma \Lambda^{2}}{5}\left\{\left[6 \frac{\partial^{3} V}{\partial x^{3}} \frac{\partial V}{\partial x}+2\left(\frac{\partial^{2} V}{\partial x^{2}}\right)^{2}\right.\right. \\
& \left.\left.+\frac{6(n-3)}{\beta} \frac{\partial^{4} V}{\partial x^{4}}\right] \varphi_{n-2}+\frac{3}{\beta} \frac{\partial^{4} V}{\partial x^{4}} \varphi_{n-4}\right\}+\cdots,
\end{aligned}
$$

where $\Lambda=\hbar^{2} \beta^{2}$ /(24m) is the characteristic quantum parameter. We note that setting $\Lambda=0$ in Eq. (6) yields Brinkman's original representation $^{20,21}$ of the classical Klein-Kramers 
equation as a recurrence relation. Now we make the time dependent perturbation expansion,

$$
\varphi_{n}=\varphi_{n}^{(0)}+\Lambda \varphi_{n}^{(1)}+\Lambda^{2} \varphi_{n}^{(2)}+\cdots
$$

in Eq. (6). Just as in the classical case, by seeking an asymptotic solution of Eq. (6) for $\varphi_{0}(x, t)$ in the powers of inverse damping $\gamma^{-1}$, the Brinkman method leads in the zeroinertia or high damping limit, $\gamma^{-1}=m / \zeta \rightarrow 0$, to the continuity equation,

$$
\frac{\partial \varphi_{0}}{\partial t}+\frac{\partial j}{\partial x}=0
$$

for the probability current

$$
j(x, t)=j^{(0)}(x, t)+\Lambda j^{(1)}(x, t)+\Lambda^{2} j^{(2)}(x, t)+\cdots,
$$

where

$$
\begin{aligned}
j^{(0)}= & \frac{1}{\gamma \beta m}\left(\frac{\partial \varphi_{0}^{(0)}}{\partial x}+\beta \varphi_{0}^{(0)} \frac{\partial V}{\partial x}\right), \\
j^{(1)}= & \frac{1}{\gamma \beta m}\left(\frac{\partial \varphi_{0}^{(1)}}{\partial x}+\beta \varphi_{0}^{(1)} \frac{\partial V}{\partial x}\right)+\frac{2}{\gamma \beta m} \frac{\partial}{\partial x}\left(\varphi_{0}^{(0)} \frac{\partial^{2}}{\partial x^{2}} V(x)\right), \\
j^{(2)}= & \frac{1}{\gamma \beta m}\left(\frac{\partial \varphi_{0}^{(2)}}{\partial x}+\beta \varphi_{0}^{(2)} \frac{\partial V}{\partial x}\right)+\frac{2}{\gamma \beta m} \frac{\partial}{\partial x}\left\{\frac{\partial^{2} V}{\partial x^{2}} \varphi_{0}^{(1)}\right. \\
& \left.-\frac{2}{5}\left[\left(\frac{\partial^{2} V}{\partial x^{2}}\right)^{2}+3 \frac{\partial^{3} V}{\partial x^{3}} \frac{\partial V}{\partial x}-\frac{3}{\beta} \frac{\partial^{4} V}{\partial x^{4}}\right] \varphi_{0}^{(0)}\right\} .
\end{aligned}
$$

Equivalently, Eq. (7) can be written as a quantum Smoluchowski equation for the configuration space distribution function $\varphi_{0}(x, t)=P(x, t)$, viz.

$$
\frac{\partial P}{\partial t}=\frac{\partial}{\partial x}\left\{\frac{P}{\zeta} \frac{\partial V}{\partial x}+\frac{\partial}{\partial x}(D P)\right\},
$$

where $D$ is the diffusion coefficient given by

$$
\begin{aligned}
D(x)= & \frac{1}{\zeta \beta}\left\{1+2 \Lambda \frac{\partial^{2} V}{\partial x^{2}}-\frac{4 \Lambda^{2}}{5}\left[\left(\frac{\partial^{2} V}{\partial x^{2}}\right)^{2}+3 \frac{\partial^{3} V}{\partial x^{3}} \frac{\partial V}{\partial x}\right.\right. \\
& \left.\left.-\frac{3}{\beta} \frac{\partial^{4} V}{\partial x^{4}}\right]+\cdots\right\} .
\end{aligned}
$$

In writing Eqs. (7) and (8), we noted that the classical functions $\varphi_{n}^{(0)}(x, t)$ all vanish for $n>0$ in the noninertial limit because the equilibrium distribution of the momenta has set in. We emphasize that Eq. (8) comprises exactly the quantum Smoluchowski equation to $o\left(\Lambda^{2}\right)$ as previously obtained ${ }^{14}$ in much simpler fashion directly from Eq. (8) adapting Einstein's method by imposing the Wigner configuration space distribution as the stationary solution rendering the probability current zero rather than the Maxwell-Boltzmann distribution, thus allowing one to determine the diffusion coefficient $D(x)$ explicitly. Here the quantum effects should become more pronounced at lower temperatures $T$, smaller mass $m$, and larger potential barriers, and should be detectable when the dimensionless quantum correction terms in Eq. (9) [e.g., $\beta \Lambda V^{\prime \prime}(x)$, etc.] are comparable with unity (however, the quantum correction parameter must not be too large in order to guarantee convergence of the perturbation expansion of $D$ in $\Lambda$ ).

\section{REPRESENTATION OF THE QUANTUM SMOLUCHOWSKI EQUATION AS A RECURRENCE RELATION}

Introducing the dimensionless variable $y=x /\left\langle x^{2}\right\rangle_{\mathrm{cl}}^{1 / 2}$ and the parameter $2 \Lambda / \beta\left\langle x^{2}\right\rangle_{\mathrm{cl}} \rightarrow \Lambda$, where $\left\langle x^{2}\right\rangle_{\mathrm{cl}}$ is the classical value $(\hbar \rightarrow 0)$ of the mean square displacement, Eq. (8) becomes

$$
\tau \frac{\partial P}{\partial t}=\frac{\partial}{\partial y}\left[P \frac{\partial V}{\partial y}+\frac{\partial}{\partial y}\left(1+\Lambda \frac{\partial^{2} V}{\partial y^{2}}+\cdots\right) P\right],
$$

where $\tau=\zeta \beta\left\langle x^{2}\right\rangle_{\mathrm{cl}}$,

$$
V(y)=\beta V(x)=A y^{2}+B y^{4},
$$

$A=\beta a\left\langle x^{2}\right\rangle_{\mathrm{cl}} / 2$, and $B=\beta b\left\langle x^{2}\right\rangle_{\mathrm{cl}}^{2} / 4$. For $A<0$ and $B>0$ (which is the case of interest, i.e., distinct double wells), the potential $V(y)$ has two minima separated by a maximum at $y=0$ with a potential barrier of $\Delta V=Q=A^{2} / 4 B$. The normalization condition $\left\langle y^{2}\right\rangle_{\mathrm{cl}}=1$ implies that the constants $A$ and $B$ are not independent and are related via ${ }^{9,10}$

$$
B(Q)=\frac{1}{8}\left[\frac{D_{-3 / 2}(-\sqrt{2 Q})}{D_{-1 / 2}(-\sqrt{2 Q})}\right]^{2},
$$

where $D_{v}(z)$ is Whitaker's parabolic cylinder function of the order of $v{ }^{19}$ For $Q \gg 1, B \sim Q$, while for small $Q$,

$$
\begin{aligned}
B= & \frac{\Gamma^{2}(3 / 4)}{\Gamma^{2}(1 / 4)}+\frac{\Gamma(3 / 4)\left[\Gamma^{2}(1 / 4)+\Gamma(-1 / 4) \Gamma(3 / 4)\right]}{\Gamma^{3}(1 / 4)} \sqrt{Q} \\
& +\cdots,
\end{aligned}
$$

where $\Gamma$ is the gamma function. ${ }^{19}$

The stationary solution of Eq. (10) is the equilibrium Wigner distribution function in configuration space given by (restricted to second order terms in $\Lambda)^{14}$

$$
\begin{aligned}
P_{\mathrm{st}}(y)= & \frac{1}{Z} e^{-\beta V(y)}\left\{1+\frac{\Lambda}{2}\left[\left(V^{\prime}(y)\right)^{2}-2 V^{\prime \prime}(y)\right]\right. \\
& +\frac{\Lambda^{2}}{40}\left[5\left(V^{\prime}(y)\right)^{4}+36\left(V^{\prime \prime}(y)\right)^{2}+48 V^{\prime \prime \prime}(y) V^{\prime}(y)\right. \\
& \left.\left.-44 V^{\prime \prime}(y)\left(V^{\prime}(y)\right)^{2}-24 V^{(4)}(y)\right]+\cdots\right\},
\end{aligned}
$$

where $Z=\int_{-\infty}^{\infty} P_{\text {st }}(y) d y$ is the quantum partition function. The partition function $Z$ can also be expressed in terms of Whitaker's functions as

$$
Z=Z_{\mathrm{cl}}\left(1+\Lambda z_{1}+\cdots\right),
$$

where $Z_{\mathrm{cl}}=\int_{-\infty}^{\infty} e^{-V(y)} d y$ is the classical partition function given by

$$
Z_{\mathrm{cl}}=\int_{-\infty}^{\infty} e^{-V(y)} d y=\sqrt{\pi}(2 B)^{-1 / 4} e^{Q / 2} D_{-1 / 2}(-\sqrt{2 Q}),
$$

and 


$$
\begin{aligned}
z_{1} & =\frac{1}{2 Z_{\mathrm{cl}}} \int_{-\infty}^{\infty}\left[V^{\prime 2}(y)-2 V^{\prime \prime}(y)\right] e^{-V(y)} d y \\
& =-\sqrt{2 B} \frac{D_{1 / 2}(-\sqrt{2 Q})+D_{-3 / 2}(-\sqrt{2 Q})}{D_{-1 / 2}(-\sqrt{2 Q})}
\end{aligned}
$$

constitutes the first order quantum correction to it.

Now, in order to calculate the quantum position correlation function

$$
C(t)=\beta^{-1}\left\langle\int_{0}^{\beta} \hat{y}(-i \lambda \hbar) \hat{y}(t) d \lambda\right\rangle_{0},
$$

from linear response theory, ${ }^{35}$ the decay transient of the system of quantum Brownian particles following instantaneous switchoff of an external constant field of small magnitude $\varepsilon$ is required. The small magnitude condition implies that, having suddenly removed the field at time $t=0$, one is solely interested in the relaxation of the system from an equilibrium state I with the potential $V(y)-\varepsilon y$ and the distribution function $P_{\mathrm{st}}^{\varepsilon}(t \leq 0)$ to a new equilibrium state II with the unperturbed potential $V(y)$ and the distribution function $P_{\mathrm{st}}(t$ $\rightarrow \infty$ ) given by Eq. (12). Using linear response theory, whereby one evaluates the decay transient directly, circumvents the difficulties associated with the symmetrization of correlation functions in quantum mechanics (cf., Ref. 35, Sec 4.4.1). The initial stationary distribution function $P_{\mathrm{st}}^{\varepsilon}$ is given in linear response by

$$
P_{\mathrm{st}}^{\varepsilon}(y) \cong P_{\mathrm{st}}(y)+\varepsilon\left[y P_{\mathrm{st}}(y)-\Lambda Z^{-1} V^{\prime}(y) e^{-V(y)}+\cdots\right] .
$$

Note that the transient response so formulated is truly linear because the change in amplitude $\varepsilon$ of the external field is infinitesimally small, $\varepsilon \rightarrow 0$.

As in the classical case, we can seek the solution of Eq. (10) for $t \geq 0$ as a series of Hermite polynomials in the po- sition variable, viz.,

$$
P(y, t)=P_{\mathrm{st}}(y)+\varepsilon \kappa e^{-\left[\kappa^{2} y^{2}+V(y)\right] / 2} \sum_{q=0}^{\infty} \frac{c_{q}(t) H_{q}(\kappa y)}{\sqrt{\pi 2^{q} q !}},
$$

where $\kappa$ is a scaling factor chosen so as to ensure optimum convergence of the continued fractions for the Fourier coefficients involved as suggested by Voigtlaender and Risken ${ }^{1}$ (all results for the observables are independent of the chosen value of $\kappa)$. The initial condition for $P(y, t)$ at $t=0$ is obviously $P(y, 0)=P_{\text {st }}^{\varepsilon}(y)$. We remark in passing that the solution in the classical case may be expressed as a hierarchy of three-term differential recurrence relations in $y^{n}$ as described in Ref. 2, Sect. 6. However, these recurrence relations $d i$ verge for the potential of greatest interest, namely, $A<0$, and so are useless for calculating the after-effect solution unlike those resulting from an orthogonal expansion of the form of Eq. (18).

By substituting Eq. (18) into Eq. (10) and noting the recurrence relations ${ }^{19}$

$$
d H_{n}(z) / d z=2 n H_{n-1}(z)
$$

and

$$
H_{n+1}(z)=2 z H_{n}(z)-2 n H_{n-1}(z),
$$

we have, after lengthy calculations, a nine term recurrence relation for the time dependent Fourier coefficients $c_{q}(t)$ restricted to the terms linear in the quantum parameter $\Lambda$,

$$
\begin{aligned}
\tau \frac{d}{d t} c_{q}(t)= & h_{q} c_{q+8}(t)+g_{q}^{+} c_{q+6}(t)+f_{q}^{+} c_{q+4}(t)+e_{q}^{+} c_{q+2}(t) \\
& +d_{q} c_{q}(t)+e_{q-2}^{-} c_{q-2}(t)+f_{q-4}^{-} c_{q-4}(t) \\
& +g_{q-6}^{-} c_{q-6}(t)+h_{q-8} c_{q-8}(t),
\end{aligned}
$$

where

$$
\begin{aligned}
d_{n}= & d_{n}^{0}+\Lambda d_{n}^{1}=-\frac{\sqrt{B}}{2 \alpha^{6}}\left[4 \sqrt{Q} \alpha^{6}+\left(\alpha^{4}+4 Q-6\right)(2 n+1) \alpha^{4}-12 \sqrt{Q} \alpha^{2}\left(2 n^{2}+2 n+1\right)+5(2 n+1)\left(2 n^{2}+2 n+3\right)\right] \\
& +\frac{\Lambda B}{\alpha^{8}}\left[2 \sqrt{Q}\left(\alpha^{4}-4 Q+12\right)(2 n+1) \alpha^{6}-3\left(2 n^{2}+2 n-1\right) \alpha^{8}+12\left(2 n^{2}+2 n+1\right)(5 Q-3) \alpha^{4}-70\left(2 n^{2}+2 n+3\right)\right. \\
& \left.\times(2 n+1) \sqrt{Q} \alpha^{2}+105\left(2 n^{4}+4 n^{3}+10 n^{2}+8 n+3\right)\right], \\
e_{n}^{ \pm}= & e_{n}^{0 \pm}+\Lambda e_{n}^{1 \pm}=\frac{\sqrt{B}}{2 \alpha^{6}} \sqrt{(n+1)(n+2)}\left\{\left[\left(\alpha^{4}-4 Q+6\right) \alpha^{4}+8 \sqrt{Q} \alpha^{2}(2 n+3)-15\left(n^{2}+3 n+3\right)\right]\right. \\
& +\frac{4 \Lambda \sqrt{B}}{\alpha^{2}}\left[-\sqrt{Q} \alpha^{10} \mp 2(2 Q-3) \alpha^{8}-4(\mp(6+4 n)+Q-3) \sqrt{Q} \alpha^{6}+\left(\mp 15\left(n^{2}+3 n+3\right)+4(2 n+3)(5 Q-3)\right)\right. \\
& \left.\left.\times \alpha^{4}-105\left(n^{2}+3 n+3\right) \sqrt{Q} \alpha^{2}+42(2 n+3)\left(n^{2}+3 n+5\right)\right]\right\},
\end{aligned}
$$




$$
\begin{aligned}
f_{n}^{ \pm}= & f_{n}^{0 \pm}+\Lambda f_{n}^{1 \pm}=-\frac{\sqrt{B}}{2 \alpha^{6}} \sqrt{(n+1)(n+2)(n+3)(n+4)}\left\{\left[-4 \sqrt{Q} \alpha^{2}+3(2 n+5)\right]-\frac{2 \Lambda \sqrt{B}}{\alpha^{2}}\left[3 \alpha^{8} \pm 16 \sqrt{Q} \alpha^{6}+4(5 Q-3) \alpha^{4}\right.\right. \\
& \left.\left.-6(2 n+5) \alpha^{2}\left(7 \sqrt{Q} \pm 2 \alpha^{2}\right)+42\left(2 n^{2}+10 n+15\right)\right]\right\}, \\
g_{n}^{ \pm}= & g_{n}^{0 \pm}+\Lambda g_{n}^{1 \pm}=-\frac{\sqrt{B}}{2 \alpha^{6}} \sqrt{(n+1)(n+2)(n+3)(n+4)(n+5)(n+6)}\left\{1-\frac{4 \Lambda \sqrt{B}}{\alpha^{2}}\left[\mp 3 \alpha^{4}-7 \sqrt{Q} \alpha^{2}+12 n+42\right]\right\}, \\
h_{n}= & \Lambda h_{n}^{1}=\frac{3 \Lambda B}{\alpha^{8}} \sqrt{(n+1)(n+2)(n+3)(n+4)(n+5)(n+6)(n+7)(n+8)},
\end{aligned}
$$

and $\alpha=\kappa / B^{1 / 4}$. Now, Eq. (19) in the usual manner of perturbation theory represents to zero order in $\Lambda$ the classical recurrence relation and also that relation forced by the quantum terms to first order in $\Lambda$. By invoking the familiar general matrix continued fraction method for solving classical recurrence relations generated by the Fokker-Planck equation, ${ }^{4,21}$ we have the solution of the differential recurrence Eq. (19) (details of this solution are given in the Appendix). Having evaluated the Fourier coefficients $c_{q}(t)$, we have the probability density $P(y, t)$ and observables such as the position correlation function, its characteristic relaxation times, dynamic susceptibility, etc.

\section{CALCULATION OF OBSERVABLES}

In transient relaxation of a system of nonlinear oscillators starting from an equilibrium state I with the distribution function $P_{\mathrm{st}}^{\varepsilon}(y)(t \leq 0)$ given by Eq. (17) to a new equilibrium state II with the distribution function $P_{\mathrm{st}}(y)(t \rightarrow \infty)$ given by Eq. (12), the averaged displacement $\langle\hat{y}\rangle(t)$ relaxes from the equilibrium value $\langle\hat{y}\rangle_{\varepsilon}$ to the value $\langle\hat{y}\rangle_{0}=0$, the transient being described by an appropriate relaxation function. Noting that the Weyl symbol of the coordinate operator $\hat{y}$ is $y,{ }^{13}$ we have via the Weyl correspondence the averaged displacement $\langle\hat{y}\rangle(t)$ following removal of the small external constant field

$$
\langle\hat{y}\rangle(t)=\int_{-\infty}^{\infty} y P(y, t) d y .
$$

By using the orthogonality properties of the Hermite polynomials, we have from Eqs. (18) and (20) the average $\langle\hat{y}\rangle(t)$ as a linear combination of the time dependent Fourier coefficients $c_{2 q-1}(t)$,

$$
\langle\hat{y}\rangle(t)=\varepsilon \frac{\alpha Z_{\mathrm{cl}} B^{1 / 4}}{\sqrt{\pi}} \sum_{q=1}^{\infty} c_{2 q-1}(0) c_{2 q-1}(t) .
$$

According to linear response theory, ${ }^{35}\langle\hat{y}\rangle(t)$ is related to the linear response after-effect function, that is the position correlation function $C(t)$ from Eq. (16), via

$$
\langle\hat{y}\rangle(t)=\varepsilon C(t) .
$$

Thus we have the position correlation function in series form directly from the after-effect solution as

$$
C(t)=\frac{\alpha Z_{\mathrm{cl}} B^{1 / 4}}{\sqrt{\pi}} \sum_{q=1}^{\infty} c_{2 q-1}(0) c_{2 q-1}(t) .
$$

The corresponding spectrum $\widetilde{C}(\omega)=\int_{0}^{\infty} C(t) e^{-i \omega t} d t$ is

$$
\widetilde{C}(\omega)=\frac{\alpha Z_{\mathrm{cl}} B^{1 / 4}}{\sqrt{\pi}} \sum_{q=1}^{\infty} c_{2 q-1}(0) \widetilde{c}_{2 q-1}(\omega)
$$

and so we have the dynamic susceptibility $\chi(\omega)=\chi^{\prime}(\omega)$ $-i \chi^{\prime \prime}(\omega)$ as ${ }^{35}$

$$
\chi(\omega)=-\int_{0}^{\infty} \frac{d}{d t} C(t) e^{-i \omega t} d t=C(0)-i \omega \tilde{C}(\omega) .
$$

Moreover, the correlation time $\tau_{\text {int }}$ [the area under the relaxation function $C(t)]$ is given by

$$
\tau_{\text {int }}=\widetilde{C}(0) / C(0) .
$$

Now, one may formally introduce another time constant characterizing the time behavior of $C(t)$. This is the effective relaxation time $\tau_{\text {ef }}$ defined by

$$
\tau_{\mathrm{ef}}=-C(0) / \dot{C}(0),
$$

yielding precise information on the initial decay of $C(t)$ in the time domain. The relaxation times $\tau_{\text {int }}$ and $\tau_{\text {ef }}$ may equivalently be defined in terms of the eigenvalues $\lambda_{k}$ of the Fokker-Planck operator $L_{\mathrm{FP}}$ because $C(t)$ can be written formally as the discrete set of relaxation modes,

$$
C(t) / C(0)=\sum_{k} a_{k} e^{-\lambda_{k} t},
$$

where $\Sigma_{k} a_{k}=1$, so that from Eqs. (26) $-(28) \tau_{\text {int }}=\Sigma_{k} a_{k} / \lambda_{k}$ and $\tau_{\mathrm{ef}}=\left(\Sigma_{k} \lambda_{k} a_{k}\right)^{-1}$. Thus the relaxation times $\tau_{\text {int }}$ and $\tau_{\mathrm{ef}}$ contain contributions from all the $\lambda_{k}$. Here, only the smallest nonvanishing eigenvalue $\lambda_{1}$, which is $\sim e^{-Q} \ll 1$ in the low temperature limit [see Eq. (34)], is associated with the slowest relaxation mode; the remaining $\lambda_{k}(k \neq 1)$ characterize highfrequency "intrawell" modes. ${ }^{4}$ The eigenvalue $\lambda_{1}$ is usually associated with the long time behavior of $C(t)($ Ref. 4) and for sufficiently high potential barriers essentially corresponds to the Kramers escape rate. ${ }^{4,21}$ Here the potential has two equivalent wells, so that the dependences of $\tau_{\text {int }}$ and $1 / \lambda_{1}$ on the model parameters are similar. 


\section{EVALUATION OF THE CHARACTERISTIC TIMES}

The correlation time $\tau_{\text {int }}$, rendered in series form by Eqs. (24) and (26), can also be obtained in closed integral form because [just as the classical case, ${ }^{4,21}$ in all systems with dynamics described by a single variable Fokker-Planck-like equation, of which Eq. (10) is a particular example], the correlation time $\tau_{\text {int }}$ of the position correlation function for the double well potential may be expressed in exact analytical form in terms of the diffusion coefficient and stationary distribution as

$\tau_{\text {int }}=\frac{\tau}{C(0)} \int_{-\infty}^{\infty} \frac{1}{D(y) P_{\mathrm{st}}(y)} \int_{-\infty}^{y} x P_{\mathrm{st}}(x) d x \int_{-\infty}^{y} \frac{\partial}{\partial \varepsilon} P_{\mathrm{st}}^{\varepsilon}(z) d z d y$,

where

$$
\begin{aligned}
& C(0)=\left\langle y^{2}\right\rangle_{0}-\Lambda+\cdots, \\
& \int_{-\infty}^{y} \frac{\partial}{\partial \varepsilon} P_{\mathrm{st}}^{\varepsilon}(z) d z=\int_{-\infty}^{y} z P_{\mathrm{st}}(z) d z+\Lambda \frac{e^{-V(y)}}{Z_{\mathrm{cl}}}+\cdots,
\end{aligned}
$$

and $\left\langle y^{2}\right\rangle_{0}=\int_{-\infty}^{\infty} y^{2} P_{\text {st }}(y) d y$. Here we noted that

$$
C(0)=\partial_{\varepsilon}\langle\hat{y}\rangle_{\varepsilon}=\int_{-\infty}^{\infty} y\left(y P_{\mathrm{st}}-\Lambda \frac{e^{-V}}{Z_{\mathrm{cl}}} \frac{\partial V}{\partial y}+\cdots\right) d y .
$$

Numerical calculation shows that the integral representation of the solution, Eq. (29), and the matrix continued fraction solution from Eqs. (24) and (26) yield the same results.

Now, the effective relaxation time $\tau_{\text {ef }}$ from Eq. (27) is

$$
\tau_{\mathrm{ef}}=-\frac{\langle\hat{y}\rangle_{\varepsilon}}{\partial_{t}\langle\hat{y}\rangle_{\varepsilon}}=\tau C(0),
$$

where we noted Eq. (30) and that

$$
\partial_{t}\langle y\rangle_{\varepsilon}=-\left\langle\partial_{y} V\right\rangle_{\varepsilon}=\int_{-\infty}^{\infty} \frac{\partial V}{\partial y}\left[y P_{\mathrm{st}}-\frac{\Lambda}{Z_{\mathrm{cl}}} e^{-V} \frac{\partial V}{\partial y}\right] d y=1 .
$$

In the classical limit, $\Lambda \rightarrow 0$, Eqs. (29) and (31) reduce to the corresponding classical results ${ }^{5}$

$$
\begin{aligned}
& \tau_{\text {int }}=\frac{\tau}{Z_{\mathrm{cl}}} \int_{-\infty}^{\infty} e^{V(y)}\left[\int_{-\infty}^{y} z e^{-V(z)} d z\right]^{2} d y, \\
& \tau_{\mathrm{ef}}=\tau .
\end{aligned}
$$

In the low temperature limit, $Q \gg 1, \lambda_{1}$ (and thus the correlation time $\tau_{\text {int }}$ ) can be estimated in a simple manner as $\lambda_{1}$ $\sim \Gamma$, where $\Gamma$ is the semiclassical Kramers escape rate in the VHD limit. As shown in Ref. 14, in the VHD limit and above the crossover temperature $T>T_{C}$ between tunneling and thermal activation, the quantum Kramers escape rate $\Gamma_{q}$ can be written as

$$
\Gamma_{q}=\Xi \Gamma_{\mathrm{cl}},
$$

where $\Gamma_{\mathrm{cl}}=\left(m \omega_{c} \omega_{a} / 2 \pi \zeta\right) e^{-\beta \Delta V}$ is the classical VHD Kramers escape rate, $\Delta V$ is a potential barrier, and $\Xi$ is Wigner's quantum transition state theory correction factor, namely, ${ }^{36}$

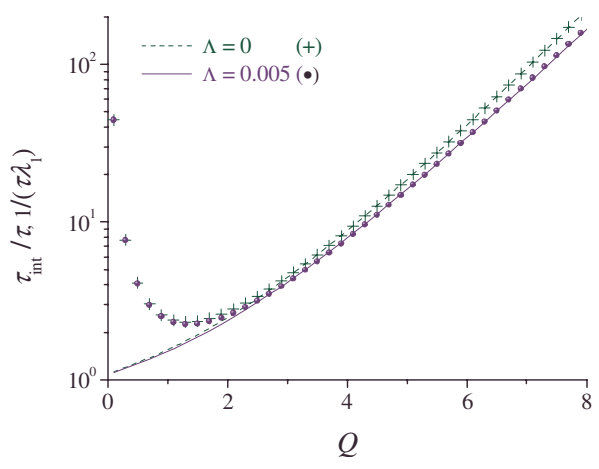

FIG. 1. Correlation time $\tau_{\text {int }} / \tau$, Eq. (29), vs the barrier parameter $Q$ for $\Lambda$ $=0$ (classical limit, dashed line) and $\Lambda=0.005 / Q$ (solid line), as compared with $1 /\left(\lambda_{1} \tau\right)$ from the asymptotic escape rate Eq. (33) (pluses and filled circles).

$$
\Xi=\frac{\omega_{c}}{\omega_{a}} \frac{\sinh \left(\hbar \beta \omega_{a} / 2\right)}{\sin \left(\hbar \beta \omega_{c} / 2\right)},
$$

where $\omega_{c}=\sqrt{\left|V^{\prime \prime}\left(x_{c}\right)\right| / m}$ and $\omega_{a}=\sqrt{V^{\prime \prime}\left(x_{a}\right) / m}$ are the barrier and the well angular frequencies [points $c$ and $a$ are the maximum and minimum of the potential $V(x)$, respectively]. In dimensionless variables, $\Xi$ becomes

$$
\Xi=\sqrt{2} \frac{\sinh \sqrt{12 \Lambda \sqrt{Q B}}}{\sin \sqrt{24 \Lambda \sqrt{Q B}}} \approx 1+6 \Lambda \sqrt{Q B}+\cdots .
$$

Furthermore, the classical VHD escape rate $\Gamma_{\mathrm{cl}}$ for the double well potential has been estimated, e.g., in Ref. 5 and is given by (in our notation)

$$
1 / \Gamma_{\mathrm{cl}} \sim \frac{\tau \pi e^{Q}}{4 \sqrt{2} Q}\left(1+\frac{5}{8 Q}+\cdots\right)
$$

The above asymptotic equations may be used to estimate $\lambda_{1}$ in the low temperature limit only. In general, $\lambda_{1}$ can be estimated via the $\mathrm{MFPT}^{37,38}$ as $\lambda_{1}^{-1}=\tau_{\mathrm{MFPT}}$, where $\tau_{\mathrm{MFPT}}$ is the MFPT for transitions from the point domain $y_{\min }$ at the bottom of a well to $y_{\max }=0$, where the potential $V(y)$ attains its maximum. Thus we have $\tau_{\text {MFPT }}$ in the closed form ${ }^{38}$

$$
\tau_{\mathrm{MFPT}}=\tau \int_{-\infty}^{0} \frac{1}{D(y) P_{\mathrm{st}}(y)} \int_{-\infty}^{y} P_{\mathrm{st}}(x) d x d y .
$$

In the high barrier approximation, $Q \gg 1$, estimation of the integrals in Eq. (35) using steepest descents yields the wellknown Larsson-Kostin asymptotic formula Eq. (34) for the escape rate.

Results of the comparison of the correlation time $\tau_{\text {int }}$ given by Eq. (29) with asymptotic estimates Eq. (33) are shown in Fig. 1. Equation (33) provides an accurate approximation to the correlation time for high barriers $(Q>3)$. As $Q$ increases, the correlation time $\tau_{\text {int }}$ and $\lambda_{1}^{-1}$ decrease; a phenomenon that can be ascribed to effective lowering of the potential barrier due to quantum tunneling, which increases the escape rate. These quantum effects manifest themselves even for very small values of $\Lambda$ because $\Lambda$ appears in products with the factor $12 \sqrt{Q B}$, which may take very large values in the low temperature limit. 


\section{LINEAR DYNAMIC SUSCEPTIBILITY}

According to Eqs. (28) and (25), the dynamic susceptibility is an infinite sum of Lorentzians, viz.,

$$
\frac{\chi(\omega)}{\chi}=\sum_{p} \frac{c_{p}}{1+i \omega / \lambda_{p}}
$$

where $\chi=C(0)$ is the static susceptibility. In the low- $(\omega$ $\rightarrow 0)$ and high- $(\omega \rightarrow \infty)$ frequency limits, the behavior of the susceptibility may easily be evaluated. We have from Eq. (36), respectively, for $\omega \rightarrow 0$ and for $\omega \rightarrow \infty$

$$
\begin{aligned}
& \chi(\omega) \approx \chi\left(1-i \omega \tau_{\mathrm{int}}+\cdots\right), \\
& \chi(\omega) \sim \chi\left(i \omega \tau_{\mathrm{ef}}\right)^{-1}+\cdots .
\end{aligned}
$$

However, as we shall see below, only two bands appear in the spectrum of $\chi^{\prime \prime}(\omega)$ in the low temperature limit $Q \gg 1$ (such behavior is typical of both classical and quantum systems ${ }^{4}$ ). The low-frequency band is due to the slowest (overbarrier) relaxation mode. The characteristic frequency and the half width of this band are determined by $\lambda_{1}$. The high-frequency band of $\chi^{\prime \prime}(\omega)$ is due to "intrawell" modes corresponding to the eigenvalues $\lambda_{k}(k \geqslant 2)$. These intrawell modes are indistinguishable in the frequency spectrum of $\chi^{\prime \prime}(\omega)$ appearing merely as a single high-frequency Lorentzian band.

Just as the classical case, ${ }^{5}$ knowledge of $\lambda_{1}$ is sufficient to accurately predict the low frequency part of $\chi(\omega)$ as well as the long time behavior of the equilibrium correlation function $C(t)$ for all values of the barrier parameter $Q$. Thus, if one is interested solely in the low frequency region $(\omega \tau$ $\leq 1$ ), where the effect of the intrawell modes may be ignored, the dynamic susceptibility $\chi(\omega)$ may be approximated as

$$
\frac{\chi(\omega)}{\chi} \approx \frac{1-\delta}{1+i \omega / \lambda_{1}}+\delta
$$

where $\delta$ is a parameter characterizing the contribution of intrawell modes, which can be evaluated as ${ }^{4} \delta \sim \tau_{W} / \tau_{\mathrm{ef}}$, where the effective relaxation time $\tau_{\mathrm{ef}}$ is given by Eq. (31) and $\tau_{W}$ is the intrawell relaxation time. For qualitative evaluation of Eq. (39) in the low temperature limit, $Q \gg 1$, one may use the simple estimate $\delta \sim[8 Q C(0)]^{-1}$.

In order to verify the single Lorentzian approximation, we plot in Fig. 2 the real and imaginary parts of $\chi(\omega)$ calculated from the matrix continued fraction solution and the approximate Eq. (39). It is apparent from Fig. 2 that no practical difference exists between the numerical solution and the single mode approximation (the maximum relative deviation between the corresponding curves does not exceed a few percent) at low frequencies. It is also apparent from Fig. 2 that due to the quantum tunneling, which increases the escape rate, the frequency of the maximum of the low frequency peak in $\chi^{\prime \prime}(\omega)$ increases. Furthermore, the quantum effects decrease at frequencies $\omega \gg \lambda_{1}$, where barrier crossing is no longer the dominant relaxation process.
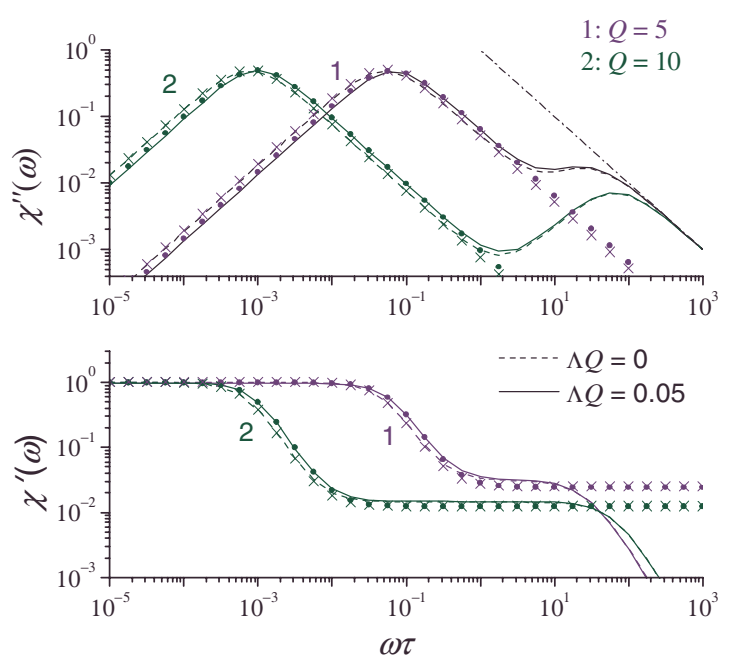

FIG. 2. Real and imaginary parts of $\chi(\omega)$ vs the normalized frequency $\tau \omega$ for $\Lambda=0$ (classical limit, dashed lines, and crosses) and $\Lambda=0.05 / Q$ (solid lines and filled circles), and barrier height $Q=5$ and $Q=10$. Solid and dashed lines are the continued fraction solution [Eq. (21) and the Appendix]. Crosses and filled circles are the single Lorentzian approximation, Eq. (39), while straight dashed-dotted lines are the high frequency asymptote Eq. (38).

\section{LINEAR STOCHASTIC RESONANCE}

A particularly interesting aspect of the Brownian motion in a double well potential is the stochastic resonance ${ }^{2}$ occurring for very high dissipation of the thermal energy of a Brownian particle to its heat bath; whereby a weak periodic forcing synchronized with the thermally activated hopping between the wells greatly enhances the rate of switching between them. Stochastic resonance is now a well-known but still remarkable effect allowing one to control the behavior of periodic signals passing through noisy systems. As a manifestation of cross coupling between stochastic and regular motions, the stochastic resonance effect is universal in physics (e.g., optics, mechanics of solids, superconductivity, surface science), communications engineering (optimal detection and tracing of signals), as well as in various branches of chemistry and biology. Comprehensive reviews of diverse aspects of stochastic resonance are available in Ref. 2. Quantum effects in the stochastic resonance have been discussed by Grifoni et $a l .{ }^{28}$

Having evaluated the dynamic susceptibility $\chi(\omega)$, one may estimate quantum effects in the signal-to-noise ratio (SNR) in stochastic resonance just as in classical case. Indeed for a weak ac driving force $f(t)=f_{0} \cos \omega t$, the SNR $\Upsilon(\omega)$ may be defined using linear response theory as ${ }^{28}$

$$
\Upsilon(\omega)=\frac{\pi}{2} \beta f_{0}^{2} \omega \frac{|\chi(\omega)|^{2}}{\chi^{\prime \prime}(\omega)}
$$

We can rewrite Eq. (40) as

$$
\Upsilon(\omega)=\frac{\beta \chi f_{0}^{2}}{\tau Q^{2}} R_{\omega}
$$

where 


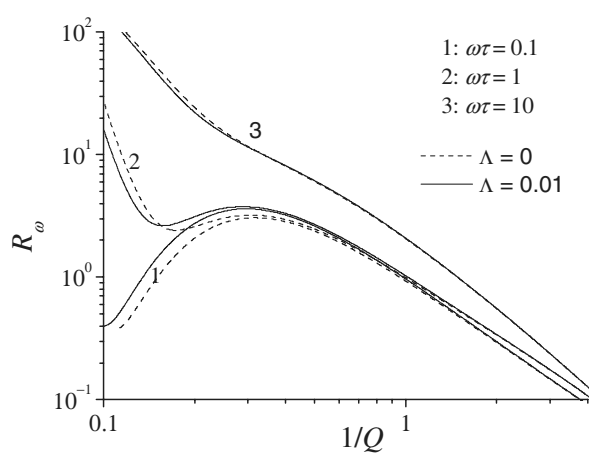

FIG. 3. SNR vs the inverse barrier height $1 / Q$ for various values of $\omega \tau$ $=0.01$ (1), 1 (2), and 10 (3). Solid lines: Eq. (42) for $\Lambda=0.005$. Dashed lines: the classical limit $(\Lambda=0)$.

$$
R_{\omega}=\frac{\pi}{2} \omega \tau Q^{2} \frac{|\chi(\omega)|^{2}}{\chi \chi^{\prime \prime}(\omega)}
$$

is the dimensionless SNR. Noting Eq. (37), we see that in the adiabatic limit $(\omega \rightarrow 0)$ the SNR is given by

$$
R_{0}=\frac{\pi \tau}{2 \tau_{\text {int }}} Q^{2},
$$

where $\tau_{\text {int }}$ is given by Eq. (29). As shown above, the time $\tau_{\text {int }}$ is very close to $1 / \lambda_{1}$ for all values of $Q \gg 1$ so that either quantity may be used for qualitative estimations.

The function $R_{\omega}$ versus the dimensionless temperature parameter $Q^{-1}$ is shown in Fig. 3. For $\omega=0$, the main maximum of $R_{0}$ is attained at $Q \sim 3$ and the quantum effects are most pronounced in the low temperature limit causing amplification of the SNR. In the limit $Q \rightarrow \infty, R_{0} \rightarrow 0$. Meanwhile, we recall that $\lambda_{1}$ is exponentially small in $Q$ for $Q$ $\gg 1$ and decreases rapidly as the system is cooled, while all other eigenvalues of the quantum Smoluchowski operator $\lambda_{k}$ have a nonexponential dependence on $Q$. Hence, at any finite $\omega$ (i.e., outside the adiabatic limit), the ratio $\omega / \lambda_{1}$ tends to infinity with decreasing temperature, $T \rightarrow 0$, even at very low frequencies since the overbarrier transition is completely frozen out. However, the Brownian particle, although confined to a particular potential well, is not completely immobilized and can still take part in intrawell motion. Thus for $\omega \neq 0$, $R_{\omega} \rightarrow$ const as $Q \rightarrow \infty$. Furthermore, for $\omega \neq 0$, the quantum effects can lead to both amplification and attenuation of the SNR. The function $R_{\omega}$ versus the dimensionless frequency

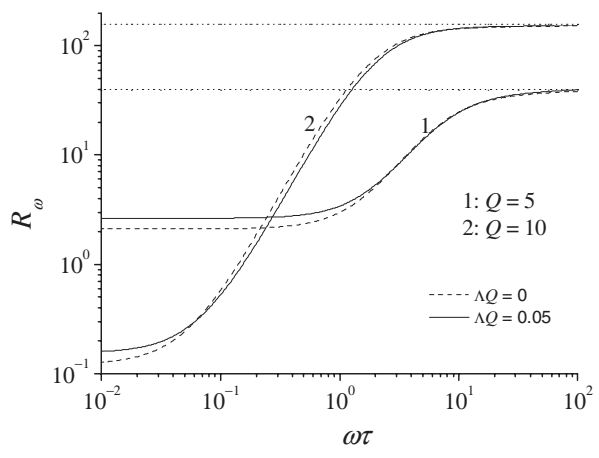

FIG. 4. SNR vs $\omega \tau$ for $Q=5$ (1) and 10 (2). Solid lines: Eq. (42) for $Q \Lambda$ $=0.05$. Dashed lines: the classical limit $(\Lambda=0)$. Dotted lines: Eq. (44). $\omega \tau$ is presented in Fig. 4 showing that the quantum effects may lead to both amplification and attenuation of the SNR. Here the SNR is a monotonically increasing function from the low frequency limit $R_{0}$ to its plateau value, viz.,

$$
R_{\omega \rightarrow \infty} \sim \pi Q^{2} /[2 C(0)] .
$$

\section{CONCLUDING REMARKS}

Thus we have shown how quantum effects in the Brownian motion of a particle in a double well potential may be studied using a semiclassical Smoluchowski equation based on the extension of Wigner's phase space formulation of quantum mechanics to an open system. The results agree with quantum reaction rate theory constituting a benchmark solution. The most significant manifestation of the quantum effects above the crossover temperature between tunneling and thermal activation appears in connection with the lowfrequency relaxation via transitions across the potential barrier. In this frequency range, the relaxation process is accurately described by a single Lorentzian with the relaxation time given by the inverse quantum rate so providing a very simple picture of the quantum relaxation. We must remark, however, that our results can be used only at low frequencies $(\omega \tau \leq 1)$ as inertial effects are completely ignored. These effects can be taken account of by considering the phase space master Eq. (3). ${ }^{24}$ Our solutions are valid only for small values of the quantum parameter $\Lambda(\Lambda \ll 1)$ since in our perturbation procedure we neglected all terms of the order of $\Lambda^{2}$ and higher. In order to improve the accuracy of calculations for larger values of $\Lambda$, additional terms of the order of $\Lambda^{2}$, etc. should be included in the Smoluchowski Eq. (8) as described in Ref. 27. Thus higher order quantum correction terms to the classical results may be calculated, in principle, to any desired degree $r$ of $\hbar^{2 r}$.

The main advantage in applying Wigner's phase-space approach to the quantum Brownian motion in a potential is that this formulation proceeds via the quantum Smoluchowski Eq. (8), which is a partial differential equation and so operators are not involved. Furthermore, the phase-space representation suggests how powerful computation techniques developed for the classical Smoluchowski equation may be extended to the quantum domain rendering a transparent treatment of quantum effects in Brownian motion. Using these techniques quantum effects on diffusive transport properties can in principle be estimated for arbitrary potentials (see, e.g., Ref. 27). We should remark that in addition to the Wigner phase space approach, the quantum Brownian motion in a potential may also be treated using a variety of other methods such as numerical simulations, ${ }^{31,39}$ the reduced density matrix, ${ }^{40,41}$ path integrals, ${ }^{16,42}$ etc. In general, these methods allow one to treat the dynamics of dissipative quantum systems. However, in spite of the progress achieved by these methods, they possess certain practical disadvantages. For example, a simple time evolution equation for the reduced density matrix does not exist. ${ }^{17}$ In spite of the formal power of numerical simulation methods, which yield solutions to many problems, the understanding and interpretation of the qualitative behavior of the relevant physical quantities 
are not always obvious. Path integral approaches for the most part have been confined to harmonic oscillator models since in general it is difficult or indeed impossible to evaluate path integrals for any other potentials. ${ }^{42}$ Wigner's phasespace approach to the quantum Brownian motion is mostly useful in the high temperature limit $T \gg T_{0}$ defined by Eq. (4). The estimations show ${ }^{27}$ that for various physical systems $T_{0}$ can be very low in comparison with the temperatures of interest, which can also very low. For example, for point Josephson junctions, which are usually treated using the model of Brownian motion in a tilted periodic potential, ${ }^{4} T$ $\sim 1.5-4 \mathrm{~K}$ and $T_{0} \sim 0.01$. For parameter ranges, where condition (4) is invalid (e.g., throughout the very-lowtemperature region, $T<T_{0}$ ) other approaches should be used. One would therefore expect that only combined use of the complementary approaches can yield a comprehensive understanding of the quantum dynamics of the Brownian particle in a potential.

\section{ACKNOWLEDGMENTS}

This publication is a result of research conducted with the financial support of the IRCSET/EGIDE Ulysses exchange program and the Royal Irish Academy mobility grant 2009. L.C. thanks IRCSET for a postgraduate scholarship.

\section{APPENDIX: MATRIX CONTINUED FRACTION SOLUTION}

The nine-term recurrence relation, Eq. (19), can be rearranged as a set of matrix three-term recurrence relations, which represent the classical recurrence relation forced by the first order quantum terms, viz.,

$$
\begin{aligned}
\tau \frac{d}{d t} \mathbf{C}_{n}(t)= & \mathbf{Q}_{n}^{0-} \mathbf{C}_{n-1}(t)+\mathbf{Q}_{n}^{0} \mathbf{C}_{n}(t)+\mathbf{Q}_{n}^{0+} \mathbf{C}_{n+1}(t) \\
& +\Lambda\left[\mathbf{Q}_{n}^{1-} \mathbf{C}_{n-1}(t)+\mathbf{Q}_{n}^{1} \mathbf{C}_{n}(t)+\mathbf{Q}_{n}^{1+} \mathbf{C}_{n+1}(t)\right]
\end{aligned}
$$

where the column vector $\mathbf{C}_{n}(t)(n \geq 1)$ is defined as

$$
\mathbf{C}_{n}(t)=\left(\begin{array}{c}
c_{8 n-7}(t) \\
c_{8 n-5}(t) \\
c_{8 n-3}(t) \\
c_{8 n-1}(t)
\end{array}\right)
$$

with $\mathbf{C}_{0}(t)=0$ and the matrices $\mathbf{Q}_{n}^{i}$ and $\mathbf{Q}_{n}^{i \pm}(i=0,1)$ given by

$$
\begin{gathered}
\mathbf{Q}_{n}^{i}=\left(\begin{array}{llll}
d_{8 n-7}^{i} & e_{8 n-7}^{i+} & f_{8 n-7}^{i+} & g_{8 n-7}^{i+} \\
e_{8 n-7}^{i-} & d_{8 n-5}^{i} & e_{8 n-5}^{i+} & f_{8 n-5}^{i+} \\
f_{8 n-7}^{i-} & e_{8 n-5}^{i-} & d_{8 n-3}^{i} & e_{8 n-3}^{i+} \\
g_{8 n-7}^{i-} & f_{8 n-5}^{i-} & e_{8 n-3}^{i-} & d_{8 n-1}^{i}
\end{array}\right), \\
\mathbf{Q}_{n}^{i+}=\left(\begin{array}{llll}
h_{8 n-7}^{i} & 0 & 0 & 0 \\
g_{8 n-5}^{i+} & h_{8 n-5}^{i} & 0 & 0 \\
f_{8 n-3}^{i+} & g_{8 n-3}^{i+} & h_{8 n-3}^{i} & 0 \\
e_{8 n-1}^{i+} & f_{8 n-1}^{i+} & g_{8 n-1}^{i+} & h_{8 n-1}^{i}
\end{array}\right),
\end{gathered}
$$

$$
\mathbf{Q}_{n}^{i-}=\left(\begin{array}{cccc}
h_{8 n-15}^{i} & g_{8 n-13}^{i-} & f_{8 n-11}^{i-} & e_{8 n-9}^{i-} \\
0 & h_{8 n-13}^{i} & g_{8 n-11}^{i-} & f_{8 n-9}^{i-} \\
0 & 0 & h_{8 n-11}^{i} & g_{8 n-9}^{i-} \\
0 & 0 & 0 & h_{8 n-9}^{i}
\end{array}\right)
$$

Next we use perturbation theory to find the solution of Eq. (A1) as

$$
\mathbf{C}_{n}(t)=\mathbf{C}_{n}^{0}(t)+\Lambda \mathbf{C}_{n}^{1}(t),
$$

treating $\Lambda$ as a small parameter. Substituting Eq. (A2) into Eq. (A1) we have in the zero order of perturbation theory the three-term recurrence relation constituting the classical solution

$$
\tau \frac{d}{d t} \mathbf{C}_{n}^{0}(t)=\mathbf{Q}_{n}^{0-} \mathbf{C}_{n-1}^{0}(t)+\mathbf{Q}_{n}^{0} \mathbf{C}_{n}^{0}(t)+\mathbf{Q}_{n}^{0+} \mathbf{C}_{n+1}^{0}(t),
$$

and in the first order of perturbation theory the forced threeterm recurrence relation

$$
\tau \frac{d}{d t} \mathbf{C}_{n}^{1}(t)=\mathbf{Q}_{n}^{0-} \mathbf{C}_{n-1}^{1}(t)+\mathbf{Q}_{n}^{0} \mathbf{C}_{n}^{1}(t)+\mathbf{Q}_{n}^{0+} \mathbf{C}_{n+1}^{1}(t)+\mathbf{R}_{n}(t),
$$

where the forcing function $\mathbf{R}_{n}(t)$ is

$$
\mathbf{R}_{n}(t)=\mathbf{Q}_{n}^{1-} \mathbf{C}_{n-1}^{0}(t)+\mathbf{Q}_{n}^{1} \mathbf{C}_{n}^{0}(t)+\mathbf{Q}_{n}^{1+} \mathbf{C}_{n+1}^{0}(t),
$$

constituting the first order quantum correction to the classical solution. The initial condition vector $\mathbf{C}_{n}(0)=\mathbf{C}_{n}^{0}(0)+\Lambda \mathbf{C}_{n}^{1}(0)$ consists of the initial values $c_{n}(0)$ with an odd value of the index $n=2 p+1$ so that we have

$$
c_{2 p+1}(0)=c_{2 p+1}^{0}(0)+\Lambda c_{2 p+1}^{1}(0),
$$

where

$$
\begin{aligned}
& c_{2 p+1}^{i}(0)= \frac{1}{Z_{\mathrm{cl}} \sqrt{2^{2 p+1}(2 p+1) !}} \int_{-\infty}^{\infty} \xi F_{i}(\xi) H_{2 p+1}(\alpha \xi) \\
& \times e^{-\left(\alpha^{2} \xi^{2}-2 \sqrt{Q} \xi^{2}+\xi^{4}\right) / 2} d \xi \\
& F_{0}(\xi)=1 / \sqrt{B}, \\
& F_{1}(\xi)=4\left[2 \xi^{2}\left(\xi^{2}-\sqrt{Q}\right)^{2}+\sqrt{Q}-3 \xi^{2}\right]+4 \sqrt{B}\left(\sqrt{Q}-\xi^{2}\right) \\
&-Z_{1} /\left(Z_{\mathrm{cl}} \sqrt{B}\right),
\end{aligned}
$$

and

$$
\begin{aligned}
\frac{Z_{1}}{\sqrt{B} Z_{\mathrm{cl}}} & =\frac{1}{\sqrt{B} Z_{\mathrm{cl}}} \int_{-\infty}^{\infty}\left[V^{\prime}\left(x^{\prime}\right)^{2}-2 V^{\prime \prime}\left(x^{\prime}\right)\right] e^{-V\left(x^{\prime}\right)} d x^{\prime} \\
& =-\sqrt{8} \frac{D_{1 / 2}(-\sqrt{2 Q})+D_{-3 / 2}(-\sqrt{2 Q})}{D_{-1 / 2}(-\sqrt{2 Q})}
\end{aligned}
$$

Now, using the one-sided Fourier transform, Eq. (A3) can be rearranged as the set of matrix three-term recurrence relations

$$
i \tau \omega \widetilde{\mathbf{C}}_{n}^{0}-\tau \mathbf{C}_{n}^{0}(0)=\mathbf{Q}_{n}^{0} \widetilde{\mathbf{C}}_{n}^{0}+\mathbf{Q}_{n}^{0+} \widetilde{\mathbf{C}}_{n+1}^{0}+\mathbf{Q}_{n}^{0-} \widetilde{\mathbf{C}}_{n-1}^{0},
$$

where $\widetilde{\mathbf{C}}_{n}^{0}=\int_{0}^{\infty} \mathbf{C}_{n}^{0}(t) e^{-i \omega t} d t$. By invoking the general method ${ }^{4}$ for solving the tridiagonal matrix recurrence relation, Eq. 
(A6), we have the zero order perturbation (i.e., classical) solution for $\widetilde{\mathbf{C}}_{1}(\omega)$ in terms of matrix continued fractions, viz.,

$$
\widetilde{\mathbf{C}}_{1}^{0}=\tau \boldsymbol{\Delta}_{1}^{0}\left\{\mathbf{C}_{1}^{0}(0)+\sum_{n=2}^{\infty}\left[\prod_{k=2}^{n} \mathbf{Q}_{k-1}^{0+} \boldsymbol{\Delta}_{k}^{0}\right] \mathbf{C}_{n}^{0}(0)\right\},
$$

where $\boldsymbol{\Delta}_{n}^{i}(i=0,1)$ are matrix continued fractions defined by the recurrence relation

$$
\boldsymbol{\Delta}_{n}^{i}=\left[i \tau \omega \mathbf{I}-\mathbf{Q}_{n}^{i}-\mathbf{Q}_{n}^{i+} \boldsymbol{\Delta}_{n+1}^{i} \mathbf{Q}_{n+1}^{i-}\right]^{-1},
$$

and $\mathbf{I}$ is the identity matrix. All other $\widetilde{\mathbf{C}}_{n}^{0}$ can be calculated from Eqs. (A6) and (A7) noting that $\widetilde{\mathbf{C}}_{0}^{0}=\mathbf{0}$. In like manner, we have the solution for the first order spectrum

$$
i \tau \omega \widetilde{\mathbf{C}}_{n}^{1}=\mathbf{Q}_{n}^{1} \widetilde{\mathbf{C}}_{n}^{1}+\mathbf{Q}_{n}^{1+} \widetilde{\mathbf{C}}_{n+1}^{1}+\mathbf{Q}_{n}^{1-} \widetilde{\mathbf{C}}_{n-1}^{1}+\tau \mathbf{C}_{n}^{1}(0)+\widetilde{\mathbf{R}}_{n},
$$

and

$$
\widetilde{\mathbf{C}}_{1}^{1}=\boldsymbol{\Delta}_{1}^{1}\left\{\tau \mathbf{C}_{1}^{0}(0)+\tilde{\mathbf{R}}_{1}+\sum_{n=2}^{\infty}\left[\prod_{k=2}^{n} \mathbf{Q}_{k-1}^{1+} \boldsymbol{\Delta}_{k}^{1}\right]\left(\tau \mathbf{C}_{n}^{0}(0)+\tilde{\mathbf{R}}_{n}\right)\right\} .
$$

All other $\widetilde{\mathbf{C}}_{n}^{1}$ can be calculated from Eqs. (A8) and (A9) noting that $\widetilde{\mathbf{C}}_{0}^{1}=\mathbf{0}$. The spectrum of the position correlation function $C(t)$ is then given by

$$
\begin{aligned}
\tilde{C}(\omega) & =\frac{\alpha Z_{\mathrm{cl}} B^{1 / 4}}{\sqrt{\pi}} \sum_{n=1}^{\infty} \mathbf{C}_{n}^{T}(0) \cdot \widetilde{\mathbf{C}}_{n} \\
& =\frac{\alpha Z_{\mathrm{cl}} B^{1 / 4}}{\sqrt{\pi}} \sum_{q=1}^{\infty} c_{2 q-1}(0) \tilde{c}_{2 q-1}(\omega),
\end{aligned}
$$

where $\tilde{c}_{2 q-1}(\omega)=\int_{0}^{\infty} c_{2 q-1}(t) e^{-i \omega t} d t$ and the symbol " $T$ " (transpose) designates transformation of a column vector $\mathbf{C}_{n}(0)$ to a row vector. The integral and effective relaxation times can be calculated via matrix continued fractions as

$$
\begin{aligned}
\tau_{\text {int }} & =\left.\frac{\alpha Z_{\mathrm{cl}} B^{1 / 4}}{\sqrt{\pi}} \sum_{n=1}^{\infty} \mathbf{C}_{n}^{T}(0) \cdot \tilde{\mathbf{C}}_{n}\right|_{\omega \rightarrow 0}, \\
\tau_{e f} & =-\left[\frac{\alpha Z_{\mathrm{cl}} B^{1 / 4}}{\sqrt{\pi}} \sum_{n=1}^{\infty} \mathbf{C}_{n}^{T}(0) \cdot \dot{\mathbf{C}}_{n}(0)\right]^{-1} .
\end{aligned}
$$

${ }^{1}$ K. Voigtlaender and H. Risken, J. Stat. Phys. 40, 397 (1985).

${ }^{2}$ L. Gammaitoni, P. Hänggi, P. Jung, and F. Marchesoni, Rev. Mod. Phys. 70, 223 (1998)

${ }^{3}$ M. Gitterman, The Noisy Oscillator (World Scientific, Singapore, 2005).

${ }^{4}$ W. T. Coffey, Yu. P. Kalmykov, and J. T. Waldron, The Langevin Equation, 2nd ed. (World Scientific, Singapore, 2004).
${ }^{5}$ Yu. P. Kalmykov, W. T. Coffey, and S. V. Titov, Physica A 377, 412 (2007).

${ }^{6}$ C. Blomberg, Physica A 86, 49 (1977).

${ }^{7}$ A. Schenzle and H. Brand, Phys. Rev. A 20, 1628 (1979).

${ }^{8}$ R. S. Larson and M. D. Kostin, J. Chem. Phys. 69, 4821 (1978); 72, 1392 (1980)

${ }^{9}$ A. Perico, R. Pratolongo, K. F. Freed, R. W. Pastor, and A. Szabo, J. Chem. Phys. 98, 564 (1993).

${ }^{10}$ Yu. P. Kalmykov, W. T. Coffey, and J. T. Waldron, J. Chem. Phys. 105, 2112 (1996)

${ }^{11}$ E. P. Wigner, Phys. Rev. 40, 749 (1932).

${ }^{12}$ Quantum Mechanics in Phase Space, edited by C. K. Zachos, D. B. Fairlie, and T. L. Curtright (World Scientific, Singapore, 2005).

${ }^{13}$ R. E. Wyatt, Quantum Dynamics with Trajectories: Introduction to Quantum Hydrodynamics (Springer, New York, 2005).

${ }^{14}$ W. T. Coffey, Yu. P. Kalmykov, S. V. Titov, and B. P. Mulligan, Phys. Chem. Chem. Phys. 9, 3361 (2007).

${ }^{15}$ W. T. Coffey, Yu. P. Kalmykov, S. V. Titov, and B. P. Mulligan, J. Phys. A: Math. Theor. 40, F91 (2007).

${ }^{16}$ J. Ankerhold, Quantum Tunneling in Complex Systems (Springer-Verlag, Berlin, 2007).

${ }^{17}$ U. Weiss, Quantum Dissipative Systems, 3rd ed. (World Scientific, Singapore, 2008).

${ }^{18}$ J. E. Moyal, Proc. Cambridge Philos. Soc. 45, 99 (1949).

${ }^{19}$ Handbook of Mathematical Functions, edited by M. Abramowitz and I. A. Stegun (Dover, New York, 1972).

${ }^{20}$ H. C. Brinkman, Physica (Amsterdam) 22, 29 (1956).

${ }^{21}$ H. Risken, The Fokker-Planck Equation, 2nd ed. (Springer-Verlag, Berlin, 1989).

${ }^{22}$ J. L. García-Palacios, Europhys. Lett. 65, 735 (2004); J. L. GarcíaPalacios and D. Zueco, J. Phys. A 37, 10735 (2004).

${ }^{23}$ W. T. Coffey, Yu. P. Kalmykov, S. V. Titov, and B. P. Mulligan, Europhys. Lett. 20011, 77 (2007); Phys. Rev. E 75, 041117 (2007).

${ }^{24}$ W. T. Coffey, Yu. P. Kalmykov, and S. V. Titov, J. Chem. Phys. 127, 074502 (2007).

${ }^{25}$ H. A. Kramers, Physica (Amsterdam) 7, 284 (1940).

${ }^{26}$ L. Machura, M. Kostur, P. Talkner, J. Łuczka, and P. Hänggi, Phys. Rev. E 73, 031105 (2006); J. Łuczka, R. Rudnici, and P. Hänggi, Physica A 351, 60 (2005); J. Dajka, L. Machura, Sz. Rogoziński, and J. Łuczka, Phys. Rev. B 76, 045337 (2007).

${ }^{27}$ W. T. Coffey, Yu. P. Kalmykov, S. V. Titov, and L. Cleary, Phys. Rev. E 78, 031114 (2008); Phys. Rev. B 79, 054507 (2009).

${ }^{28}$ M. Grifoni, L. Hartmann, S. Berchtold, and P. Hänggi, Phys. Rev. E 53, 5890 (1996).

${ }^{29}$ V. Peano and M. Thorwart, Chem. Phys. 322, 135 (2006).

${ }^{30}$ A. Igarashi and H. Yamada, Phys. Status Solidi C 1, 2812 (2004).

${ }^{31}$ M. Topaler and N. Makri, J. Chem. Phys. 101, 7500 (1994); N. Makri, J. Math. Phys. 36, 2430 (1995).

${ }^{32}$ D. Barik, B. C. Bag, and D. S. Ray, J. Chem. Phys. 119, 12973 (2003).

${ }^{33}$ S. Zhang and E. Pollak, J. Chem. Phys. 118, 4357 (2003).

${ }^{34}$ H. Grabert, Chem. Phys. 322, 160 (2006).

${ }^{35}$ R. Kubo, M. Toda, and N. Hashitsume, Statistical Physics II, Nonequilibrium Statistical Mechanics (Springer-Verlag, Berlin, Heidelberg, 1985).

${ }^{36}$ V. A. Benderskii, D. E. Makarov, and C. A. Wright, Adv. Chem. Phys. 88, 1 (1994).

${ }^{37}$ P. Hänggi, P. Talkner, and M. Borkovec, Rev. Mod. Phys. 62, 251 (1990).

${ }^{38}$ W. T. Coffey, Adv. Chem. Phys. 103, 259 (1998).

${ }^{39}$ C. H. Mac and R. Egger, Adv. Chem. Phys. 93, 39 (1995).

${ }^{40}$ A. G. Redfield, IBM J. Res. Dev. 1, 19 (1957).

${ }^{41}$ Y. J. Yan, F. Shuang, R. Xu, J. Cheng, X. Q. Li, C. Yang, and H. Zhang, J. Chem. Phys. 113, 2068 (2000).

${ }^{42}$ H. Grabert, P. Schramm, and G. L. Ingold, Phys. Rep. 168, 115 (1988). 